nodules of three cubic centimetres each and the prolapse has never since recurred, while the incontinence was very considerably improved. As, however, he still occasionally soiled his linen at night I decided to narrow the enlarged anus, which I did by a plastic procedure similar to Tait's flap-splitting operation for the repair of an incomplete laceration of the female perineum and this operation, performed 12 weeks ago, has resulted in complete relief. The boy is still under observation and his general condition has considerably improved since he acquired full control over his motions. An interesting point in this case was that when the patient was under the anæsthetic on the last occasion, before commencing the plastic operation I attempted to draw the former prolapse forcibly down but could not do so.

Manchester.

\section{THE THERAPEUTICS OF THE ICHTHYOL COMPOUNDS : WITH SPECIAL REFER- ENCE TO ICHTHOFORM AND ICHTHARGAN. ${ }^{1}$}

BY JAMES BURNET, M.A., M.B., M.R.C.P. EDIN., SENIOR CLINICAL TUTOR, EXTRAMURAI MEDICAL WARDS, ROYAI INFIRMARX, EDINRURGH; PHYSICIAN TO THE MARSHALL STREET DISPENSARY, EDINBURGH.

EXACTLY a year ago I had the thonour and privilege of bringing before your notice my experiences with ichthyol in the treatment of pulmonary diseases. ${ }^{2}$ When I was asked to give you something on the same lines this session I thought I could not do better than follow up my former paper by saying a few words about the various compounds of ichthyol. As some of you may know, I have devoted much time and careful research to the investigation of ichthyol and its preparations, so convinced am $I$ that in it we possess a most valuable therapeutic agent the properties of which depend not so much on its containing a fairly large amount of sulphur in its composition as on its being a very powerful anti-inflammatory agent. This fact I have tried to bring to the notice of the profession in a short paper on the subject which I recently contributed to the Journa of Tuberculosis. ${ }^{3}$ I may, perhaps, be allowed to quote the concluding sentences of that paper: "I am no believer in the theory that ichthyol owes its action to its antiseptic property nor in the view that its beneficial effects are entirely due to its action upon nutrition. We must go further afield and regard ichthyol in its true light as a vaso-constrictor and as an anti-inflammatory agent. This is its true action and the only one which is of much value in the application of the remedy to cases of pulmonary tuberculosis. Thus regarded ichthyol becomes a remedy of the greatest importance and one worthy the support of the profession everywhere." Ichthyol, I need hardly remind you, is a natural product that has been chemically treated. It is, in fact, a hydrocarbon oil obtained from a fossiliferous mineral, and this, after being acted on by sulphuric acid, gives us ichthyol-sulphonic acid. It is the ammonia salt of this acid which is known commercially as ichthyol. The history of the discovery of this hydrocarbon oil is of the greatest possible interest but it is somewhat outside the scope of the present paper.

Ichthyol, moreover, may be combined with quite a variety of active therapeutic substances. Thus we have ironichthyol, otherwise known as ferrichthol, calcium-ichthyol, sodium-ichtbyol, and combinations with lithium, zinc, and mercury. Lastly, we have two quite recent ichthyol compounds-namely, ichthoform and ichthargan-the former being a compound of ichthyol with formic aldehyde and the latter the silver salt of ichthyol. Although I have had considerable experience with all of these time will not permit of my making more than a passing reference to most of them, reserving the greater part of my paper to a more detailed consideration of the two last named-ichthoform and ichthargan.

Iron-ichthyol or ferrichthol is a brownish-black, amorphous

1 A paper read before the Therapeutical Society on Feb. 23rd, 1904.

2 A paper read before the Therapeutical Society on Feb. 17th, 1903, ard published in THE LANCET of August 8th, 1903, p. 384 3 October, 1903 . powder, very light and non-hygroscopic. It is practically odonrless and tasteless and is best administered in cachet or in tablet form. The amount of iron contained in this preparation is about 3.5 per cent. Aufrecht of Berlin was one of the first to publish results of clinical tests with this compound. Personal clinical experience with ferrichthol shows that it is an excellent remedy in secondary anæmia. Thus in a case of subacute rheumatism in which the red corpuscles and the hæmoglobin were reduced they returned to normal within a month under the administration of 30 grains of ferrichthol per day. Another case in which this remedy proved valuable was that of a woman suffering from carcinoma ventriculi. She was able to take ten grains of ferrichthol in the 24 hours without producing nausea. although many other iron preparations previously given had not been retained. In a case of chronic renal disease where the patient suffered from intense headache and was markedly anæmic ferrichthol proved very useful not only in improving the condition of the blood but also in diminishing the frequency and severity of the cephalic pain. In severe cases of dysmenorrhcea associated with constipation and anæmia one of the most suitable remedies is ferrichthol in ten-grain doses thrice daily. In cases of chlorosis, on the other hand, I have seen no good results follow its per:istent employment. It is, therefore, in secondary aræmia that it is of most value, and here no doubt the action of the iron is largely aided by the presence of the ichthyol with which it is combined. Although I have no personal experience of its use in skin affections it is interesting to note that Unna ${ }^{4}$ advises its adoption in all chronic conditions, especially in urticaria and analogous skin diseases, in papular and bullous affections, and in lichen as it occurs in children. Again he finds it of service in diseases complicated either by chlorosis or by anæmia. Thus in eczemas occurring in anæmic subjects the ichthyol in combination with iron improves the circulation and so does good. No doubt iron-ichthyol has a wide sphere of therapeutic application in all diseased conditions associated with anæmia, and my own observations on its value in general diseases are fully borne out by Unna's with regard to dermatolr gical conditions.

Sodium-ichthyol is a solid preparation and thus forms an excellent substitute for the internal administration of the ammonia salt as it can readily be made into pills. I am of opinion, however, that it is not nearly so active in pulmonary disease as is the original ammonia salt, though it is perhaps a little more readily taken by fastidious patients. In chronic gastric catarrh it has the power of checking the fermentative processes which form such a troublesome element in many of these cases. Generally speaking, where ichthyol is indicated this preparation should be prescribed, except in cases of pulmonary disease in which the original ammonia salt alone should be employed.

Calcium.ichthyol is well suited for use in dermatological practice and I have found it readily taken by children. It is not, however, in my experience such a generally useful salt as are some of the others. The zinc salt finds many indications for its use in diseases of the skin and hence its value appeals most to dermatologists. It may be used as an ingredient in ointments and in certain cases is to be preferred to the ammonia salt. Of the lithium salt I cannot now speak as, not being an advocate for the use of lithium, I have not felt it my duty to experiment much with this compound of ichthyol.

Within the last few years ichthyol has been made use of as a vehicle for certain insoluble agents such as cresol, eucalyptol, and even iodine. I have elsewhere fully recorded my experience of the use of iodine-anytol, as this new combination is called, and I need not stay to consider these quite recent additions to our pharmaceutical armamentarium. Of all preparations of ichthyol two stand out preeminently-namely, ichthoform and ichthargan. Of these I have been making trial for some time past and it is with the greatest possible pleasure that I take this opportunity of bringing the results of my work in this connexion before your notice.

Ichthoform is an amorphons, brownish-black powder, being practically odourless and tasteless. It is insoluble in ordinary media such as water, alcohol, and glycerine. When brought into contact with dilute alkalies it gradually

4 Monatshefte für Praktische Dermatologie, 1901, Band xxxii., Heft 5 .

5 The Medical Times, Dec. 20th, 1902 
splits up into its two components-ichthyol and formic aldehyde. The latter being a powerful germicide and the former an active anti-inflammatory agent, ichthoform is at once an antiseptic and an astringent. It is practically a non-toxic substance and can therefore be administered internally with comparative safety. Rabow and Galli-Valerio of Lausanne ${ }^{8}$ made a thorough investigation into the properties of ichthoform for the purpose of clearing up the following questions :-1. What is its influence on the development of bacteria and fungi? 2. What is its value as a deodorant? 3. How is it borne by animals? 4. What effect has it on man? They found that gelatin plates infected with such organisms as bacterium coli, bacillus typhosus, bacillus icteroides, bacillus pyocyaneus, staphylococcus pyogenes aureus, and aspergillus niger when dusted over with ichthoform showed sterility during the first week, while vigorous development took place in control plates. In some cases development occurred during the second week, but the colonies were few in number with the exception of aspergillus niger. To show the dcodorant action of ichthoform they took nine grammes of rabbit's fæces, diluted these with water, and then added half a gramme of ichthoform. This was then heated to a temperature of $37^{\circ} \mathrm{C}$., but no disagreeable odour was felt even after the lapse of several days. They then took frogs weighing 32 grammes and introduced into the abdomen one-tenth of a gramme of jchthoform withont producing any untoward result. They further experimented with guinea-pigs weighing 250 grammes and into these they injected half a gramme of ichthoform without producing any functional disturbance. As regards its effect on man it was found that from two to three grammes could be taken daily without harmful effects. It gave excellent results in in. testinal diseases and also in the treatment of wounds, in the latter case forming a valuable substitute for other less agreeable substances such as iodoform.

Personally I have used ichthoform more or less extensively in four different directions: (1) as an intestinal astringent and antiseptic; (2) as a dusting powder; (3) as an ingredient in ointments ; and (4) as a means of impregnating gauze.

1. As an intestinal astringent and antiseptic I have employed ichthoform in a large number of cases. In infantile diarrhœa, so prevalent in the summer months and no doubt due to the presence of micro-organisms, ichthoform has afforded me excellent results. My practice is to administer it to infants one year of age in doses of two grains every six hours in a little jelly. For children generally the dose ranges from two to ten grains given thrice daily. To them it may be given concealed in milk, cocoa, or gruel; but I much prefer to give it sandwiched between two thin pieces of buttered bread. In tuberculous lesions of the intestinal tract ichthoform has given me great satisfaction. Where diarrhcea is a prominent symptom I have ordered ichthoform in doses of from 10 to 15 grains thrice daily with marked $\epsilon$ ffect. In a few instances the dose has been increased to 20,25 , and even to 30 grains, but these doses are somewhat excessive and may give rise to dryness of the throat and other troublesome phenomena. These I have not observed personally in my cwn cases, but Schäfer of Munich mentions ${ }^{7}$ especially dryness of the throat as resulting in one or two of his cases. I am of opinion that these undesirable symptoms were entirely due to the remedy being given in somewhat too large doses. As he states, however, ichthoform is non-poisonous and may be given in amounts even up to ten grammes a day without any harmful effects, and so too much importance need not be attached to the occurrence of these untoward results. In mild cases I have noticed great improvement even on the third day after its administration, and within a week, as a rule, the diarrhca was found to have ceased. In more obstinate and severe cases not infrequently the dose of ichthoform has had to be raised to 30 grains before any evident effect was produced. In one or two cases I have tried rectal injections of mucilage of starch containing from 40 to 50 grains of ichthoform. As these were cases in which the patients were practically moribund no inference of any practical importance can be drawn as to the ultimate result of this form of treatment, but certainly in all cases the diarrhoea and abdominal discomfort were con. siderably alleviated. I would urge, however, that this means of employing ichthoform should be given a more extended trial.
Polacco of Milan has given us a short account ${ }^{8}$ of his experiences with ichthoform in the treatment of enteric fever. He emplojed doses of half a gramme frequently repeated, so that in some cases the patients received as much as six grammes per day. This form of treatment was at times combined with ichthyol baths. 60 grammes of ichthyol were dissolved in a bath of hot water at a temperature of $95^{\circ} \mathrm{F}$., and while the patient was in the bath this was reduced to $86^{\circ}$ and even to $81 \cdot 5^{\circ}$. The patient remained in the bath from 10 to 15 minutes and all the time an ice.bag was kept to the head. This combined method of treating enteric fever resulted in a reduction of the patient's temperature and a diminution in the frequency of the pulse, while the resuirations became fuller and deeper. Polacco's statistics for the year 1900-01 give a mortality of 5.4 per cent. under this treatment, whereas previously it had been as high as $22 \cdot 22$ per cent.

2. I shall now pass on to consider my sfcond use of ichthoform-viz, as a dusting powder. It is more particularly in cases of varicose ulcer that I have found benefit from this use of ichthoform. In many of these cases I have used it pure. Even foul discharging ulcers have healed under the simple daily free application of this powder. The fœtor rapidly disappears and the astringent action of the remedy soon makes itself felt. It thus stimulates healing by formation of healthy tissue. For foul ulcers I know no more trustworthy remedy. Admixed with equal parts of boric acid ichthoform will be found serviceable as a dusting powder for general use. Wherever there is a tendency to the formation of redundant granulation tissue during the process of wound-healing the area should be freely covered with ichthoform and zinc oxide in equal parts. For dysidrosis I find nothing so satisfactory as a powder composed of ichthoform, bismuth, and starch.

3. As an ingredient in ointments ichthoform is of special value. Time after time cases of chronic eczema have yielded to ointments into the composition of which ichtho. form has entered. It may be used in strengths of from 1 to 5 per cent. with lanoline or vaseline as hase. If used in too great strength it is apt to harden the skin and so delay the process of repair. Although, naturally, I have not had very great facilities for treating skin diseases with ichthoform. still in the few that have come under my care $I$ have made use of ichthoform with definite results. It is of service not. only in subacute and chronic eczema, but also in the impetiginous eczema so frequently met with in school children, in certain affections of the nails, and also in scabies.

4. Lastly, I have suggested its use in the form of ichthoform gauze, to be used as a substitute for iodoform gauze for packing cavities. Thus for cavities left after removing tuberculous cervical glands or in cases of ischiorectal abscess and fistula in ano nothing will be found so snitable and so $\mathrm{efficacious}$ as gauze thoroughly impregnated with ichthoform. I trust that a gauze of this nature will soon be made available for general use as a better and less expensive material than the present iodoform gauze.

If I were to sum up the conclusions which I have arrived at after considerable experience in the use of ichthoform in private and other practice I should say that: 1 . It is one of the most efficacious antiseptics which we at present possess, more especially in cases of intestinal disease where its action. is more certain and less harmful than salol and similar substances. 2. It forms an excellent substitute for iodoform in all cases where the latter is indicated. It is odourless and practically non-toxic, epithets which cannot be applied to. iodoform. 3. For internal admini-tration it is best given in small doses frequently repeated. Large single doses do not. give the same satisfactory results and are more likely to produce untoward symptoms. As much as two drachms may be given in one day if divided into small, frequently repeated doses.

Although my experience with ichthargan is perhaps hardly so extensive as with ichthoform, still I hope to be able to place before you ceitain facts of practical interest and importance which I have noted during my employment of ichthargan. Ichthargan is a brownish-black, amorphous powder, containing 28.7 per cent. of silver, and is quite odourless and tasteless. It is readily soluble in warm or cold water, in dilute alcohol, and in glycerine. It is, however, insoluble in absolute alcohol, in ether, and in chloroform. Its solutions are clear when freshly prepared but become dark on exposure to light. It is relatively non-toxic. 
Solutions should always be prepared fresh with sterilised water and filtered.

As regards the bacteriological properties of ichthargan Aufrecht has shown ${ }^{9}$ that it is more powerful than silver nitrate in its action on the streptococcus, staphylococcus pyogenes aureus, on the bacilli of enteric fever and of diphtheria, and on gonococci. He compared its effects with those produced by collargol and protargol. He not only observed the superiority of ichthargan over these other sitver componnds but he also noted that ichthargan possessed greater penetrating powers. Pieces of liver were placed in a 5 per cent. solution of ichthargan for 16 hours and it was found that the entire mass had been acted on, whereas pieces similarly treated with silver nitrate only showed a deposit of silver on their surface at the expiry of the test. It was further found that ichthargan was less poisonous than other silver salts. The lethal dose for guinea-pigs and rabbits was determined by experiment to be between 0.1 and 0.15 gramme per kilogramme of body weight, whereas that of silver nitrate was 0.015 gramme per kilogramme, and that of protargol somewhere between 002 and 0.04 per kilogramme. Aufrecht's further experiments regarding the effects of intravenous injections in animals I pass over as I fear we may fall into error if we apply too dogmatically to the human organism the results obtained in the case of the lower creation. The great fact which we must constantly bear in mind regarding ichthargan is that more than two thirds of it is made up of ichthyol and hence it combines the therapeutic action of a silver salt with the valuable analgesic, antiseptic, and anti-inflammatory effects of the former. Moreover, it contains a very large proportion of silver and is exceedingly stable in solution and, above all, is comparatively non-irritating.

On the whole I think it will be more profitable for us to consider together the various clinical uses to which ichthargan may be put rather than to pursue further its pharmacological and bacteriological properties. The therapeutic uses of ichthargan may be classified under seven different categories and we shall now look at each of these very briefly.

1. The employment of ichthargan in gonorrhoal urethritis. - Much attention has been paid by continental clinicians to the use of ichthargan in this somewhat troublesome affection, notably by Lohnstein, ${ }^{10}$ Fürst, ${ }^{11}$ Leistikow, ${ }^{12}$ Duhot, ${ }^{10}$ Goldberg. ${ }^{14}$ Rudolf, ${ }^{15}$ and others. My experience of its value in gonorrhoea has been chiefly amongst dispensary patients. These in some respects are a difficult class to deal with, a they not only lack the ordinary habits of personal cleanliness but they are apt to treat the condition somewhat lightly and to regard themselves as cured as soon as they are free from pain and genito-urinary discomfort. Again, many of them fail to return at regular intervals, while some make only one visit and nothing more is heard of them. Putting aside the cases where the visits were either too few or irregular to be of any clinical value I find there still remain 21 in which ichthargan was employed with more or less success. The mode of treatment adopted was to prescribe an alkaline diuretic mixture combined with a little tincture of hyoscyamus and to order morning and evening urethral injections of ichthargan in distilled water. The strength of solution employed varied from 1 in 5000 to 1 in 500 according as the case was acute or chronic in its nature. I found it always advisable to begin with comparatively weak solutions and gradually to increase the strength of the injection as I found the patient's urethral mucous membrane would tolerate it. In the majority of acute cases cure was effected within three weeks, the injection being employed, as a rule, thrice daily. In two subacute cases an injection of 1 in 1000 produced considerable irritation. Patients were always advised to continue the injection at bed time for some days after they were considered cured. In chronic cases, perhaps more so than in any others, were ichthargan injections found very valuable. The strength used never exceeded 1 in 500 as this was found to be sufficiently energetic. Leistikow, however, mentions much stronger solutions, but these $I$ have found from experience are apt to cause the patient considerable suffering. They

9 Deutsche Medicinische Wochenschrift, 1900 No, 31

10 Allgemeine Medicinische Central-Zeitung, 1900. No. 80

11 Deutsche Maricinische Worhenschrift. 1971, No. 14

12 Monatshefte für Praktische Dermatolngie. 19r1. No. 23.

13 Annales de la Policlinique Centrale de Bruxelles. November, 1901 15 Wherapeutische Monatshefte, March, 1902. may, indeed, have somewhat of a caustic action and so produce cicatricial contraction of the urethral canal, a most undesirable result. In the treatment of chronic urethritis I believe bougies containing a small amount of ichthargan (say, a quarter of a grain) would be more advantageous even than injections, as they are not only more readily applied to the diseased parts but they are also more penetrating in their effects. Still, even with simple injections I have had excellent results, only four out of my total of 21 cases having failed to be cured completely, although even these exceptional cases derived much benefit from ichthargan. Indeed, I fear their failure to respond to its action with the best results was entirely due to irregularity in habits, alcoholic and otherwise. It is only fair, however, to warn against the use of too strong injections, as although ichthargan is a comparatively innocuous remedy, still it has undoubtedly a caustic effect if used in greater strengths of solution than those already mentioned.

2. Ichthargan in gynacological affections.-All of you know how valuable ichthyol is in gynæcological practice and its combination with silver in the form of ichthargan is equally beneficial. The cases in which I employed it were mostly those of leucorrhcea associated with a variety of conditions, such as pelvic cellulitis, endometritis, uterine retroflexion, and cervical catarrh. My method of application was by means of pessaries containing half or one grain of active ingredient made into a mass with oil of theobroma. One of these was placed in the posterior vaginal fornix every alternate night and this was followed by boric acid douches next day aided by saline aperients. Improvement was always manifested even after the first or second pessary had been inserted and treatment rarely required to be continued for more than a few weeks. The milder cases were cured within a week; the more severe cases necessitated assiduous application of the remedy for a month or six weeks. Instead of the pessaries a 10 per cent. solution of ichthargan in glycerine may be employed. Tampons of cotton-wool are soaked in this and placed in the vagina. I prefer, however, to use pessaries, as they are cleanlier and more effective in their results. The indication for the use of ichthargan in gynæcological practice is the necessity for an astringent plus an anti-inflammatory agent.

3. Ichthargan in dermatological practice. - In the treatment of skin diseases ichthargan will be found of service when employed in the form of ointments of varying strengths. Here again my dispensary experience has proved of great use. Cases of skin affection which otherwise I should not have bad an opportunity of treating have time and again presented themselves and I need hardly say that I have lost no opportunity in experimenting with ichthargan wherever its employment seemed indicated. The ointments used varied in strength from 0.5 to 5 per cent. in equal parts of lanoline and vaseline. The stronger ointments are apt to canse much burning where the skin is at all tender or raw and for all ordinary cases a 1 or 2 per cent. ointment is quite powerful enough. My cases numbered 39 in all, a small number of cases certainly but sufficiently varied in character to afford practical evidence as to the real value of ichthargan. They include two cases of tinea tonsurans, seven of seborrbœic eczema, three of herpes zoster, and six of various types of eczema, the remainder including ulcers, boils, and $\mathrm{A}$ variety of minor skin affections. Boils after being thoroughly treated by means of inunctions of a 10 per cent. ichthargan cintment are very frequently aborted. Ringworm affecting the scalp also requires the application of a 5 or even a 10 per cent. ointment. Otherwise I think a 1 per cent. ointment is the most generally useful.

Unna ${ }^{16}$ has recorded his experience of this remedy in cases of ulceration. He used it as a dusting powder in strengths of 1 and 5 per cent. diluted with talc. He combined this with zinc gelatin bandages and compresses and obtained good results. He states that ichthargan is a keratoplastic remedy, inducing the formation of new epithelium. His best results were obtained in old callous ulcers where the horny epithelium was first removed by the application of salicylji-creasote or salicylic-cannabis plaster mulls. He also observes that the antiseptic and keratoplastic properties of ichthargan suggest its use in cases of eczema, especially as it is met with on children's heads.

4. Ichtliargan in affections of the throat and nose.- So far as I am aware the only literature on the use of ichthargan in

16 Monatshefte für Praktische Dermatologie, 1901, No. 2. 
these diseases is a paper which appeared in the Laryngoscope $e^{17}$ from the pen of Dr. B. Douglas of New York. I shall not attempt to do more here than to summarise his results, as my experience of ichthargan in this connexion is a very limited one. For nose and throat work a strength of 4 per cent. will be found most generally useful. In 10 per cent. solutions this remedy produces a disagreeable taste and nasal irritation which lasts from 15 minutes to two hours and causes some congestion of the mucous membrane, with profuse nasal discharge, lacrymation, and a certain amount of sneezing. After much careful experimenting with this new remedy Dr. Douglas found that the strength of solution generally most satisfactory was a 4 per cent. one. This usually caused slight burning and smarting but no congestion. If congestion was present at the time of application the surface soon became pale and contracted. Its action was prompt and lasted for from 15 to 20 minutes. In atrophic rhinitis it acts as a deodorant. It has also anti-inflammatory and stimulant effects and is a powerful modifier of nasal secretion. It is therefore par excellence the remedy for atrophic rhinitis. In hypertrophic cases the best results are got from a 4 or 5 per cent. solution. One of my own cases, that of a girl suffering from atrophic rhinitis, showed marked improvement after the use of a 1 per cent. ichthargan ointment. For syphilitic, tuberculous, and simple chronic laryngitis I have found pigments of a 5 per cent. ichthargan solution extremely useful.

5. Ichthargan in diseases of the eye.-Save of the value of a 0.5 per cent. ointment in cases of marginal blepharitis I cannot speak from personal experience of ichthargan as a remedy of importance in this connexion. Fortunately, however, for my present purpose I am able to quote from the experience of others better qualified to speak on this matter than myself. Thus Guttmann of Breslau has reported ${ }^{18}$ its use in $6 \mathrm{~L}$ cases of eye affections. These include 25 cases of acute catarrhal conjunctivitis, 13 of chronic conjunctivitis, seven resulting from affections of the lacrymal apparatus, ten of trachoma, six of ophthalmia neonatorum, and one of serpiginous ulcer of the cornea. His conclusions may be summarised as follows. In congestive catarrh it proves eficient although it cannot be said to replace the older remedies for such conditions. He recommends that further experiments should be made regarding its use in ophthalmia neonatorum. In affections of the lacrymal apparatus it is a very useful remover of purulent secretion. Its chief action, however, is developed in the dissecting forms of trachoma and it is one of the most useful remedies we at present possess for the medicinal treatment of pannus.

Marczel Falta, ${ }^{19}$ writing of his use of ichthargan in cases of trachoma, says that he has used it in all forms and at all stages of this disease. He found that fresh cases were completely cured by ichthargan in from six to eight weeks. In chronic cases the pannus is reduced very speedily. $\mathrm{He}$ began with a 0.5 per cent. solution and gradually increased the strength to 3 per cent. These solutions are brushed over the affected part and by this means a pannus showed retrogressive metamorphosis ("Rückbildung") in from three to four days, and a case of recent trachoma could be cured in from six to eight weeks. Similar results were obtained by Gortaloff ${ }^{20}$ in cases of trachoma, who therefore considers ichthargan an excellent remedy for the treatment of trachoma and its complications.

6. Its internal employment.-During the course of his investigations Aufrecht took five grains of ichthargan on three successive days and eight grains on the fourth. No loss of appetite was produced, the urice remained clear and free from albumin, while the stools were unaltered in appearance. This preparation may, therefore, safely be given internally. It may be given in solution in the proportion of from half a grain to two grains dissolved in eight ounces of water and of this a teaspoonful may be taken three or four times a day. It would seem useful in promoting the healing of gastric ulcers. It might also be given as an enema in cases of dysenteric and other intestinal lesions. Not having as yet tried ichthargan in the manner suggested I offer these remaris for what they are worth. Doubtless, further experience will convince us of their accuracy.

7. Ichthargan in veterinary practice. -This is, to me, a subject of much interest and I have gone through a vast collection of German literature in the hope of being able to

17 May, 1902.

18 Woshensolrift fïr Therapie und Hygiene des Anges, vol. v., No. 36. 20 Allgemeine Medicinische Central-Zeitung, 1902, No. 5. place before you a review of the work that has been done abroad in this wide field. Time, however, forbids more than a passing reference. I wish particularly to mention the names of Eugen Bass, Eberhardt, and Leibert in this connexion. Eberhardt employed intravenous injections of ichthargan solution in cases of influenza in horses. The strength used for injection was 1 per cent. in distilled water. His results were such that frequently the temperature fell to normal within two days. This has suggested a similar use of ichthargan in cases of septicæmia as it occurs in man. I wish there was time to go more fully into this matter, as it is one of great interest, but I must not detain you longer.

From what I have said it will be evident that the various preparations of ichthyol have a wide sphere of therapentic application. This remark applies especially perhaps to ichthoform and ichthargan which, after the parent remedy itself, are, in my opinion, two of the most valuable antiinflammatory agents at present in our possession. They are certainly trustworthy in their action and therefore valuable additions to our therapeutic stock. They assuredly deserve to be used more extensively by the profession in this country and if these somewhat brief remarks lead to an awakened interest in the ichthyol compounds I shall feel more than rewarded for the trouble I have taken in bringing them before you.

Edinburgh.

\section{A CASE OF METRITIS AND CELLULITIS IN THE PUERPERIUM TREATED WITH ANTISTREPTOCOCCIC SERUM.}

By JOHN W. DUNOAN, M.B., CH.B. ABERD.

THE patient was a primipara, aged 24 years. The labour was normal after the case came uncer my care, except that the membranes were at the beginuing bound to the uterus by slight adbesions that had to be broken before the os commenced to dilate. There was a history of irritation and discharge from the vulva before labour. The woman had been told by her mother and the nurse to bear down strongly and she had done so for a good while before the time when I first saw her, which was about 7 A.M. on Oct. 8th. Delivery took place the same day at 2 P.M. The perineum externally was intact but some abrasions were found in front afterwards. In the evening the fundus uteri was two fingers' breadth below the umbilicus and half a finger's breadth to the right side. The daily temperature and pulse-rate are shown on the accompanying chart. On the 9th the patient seemed to be doirg very well;

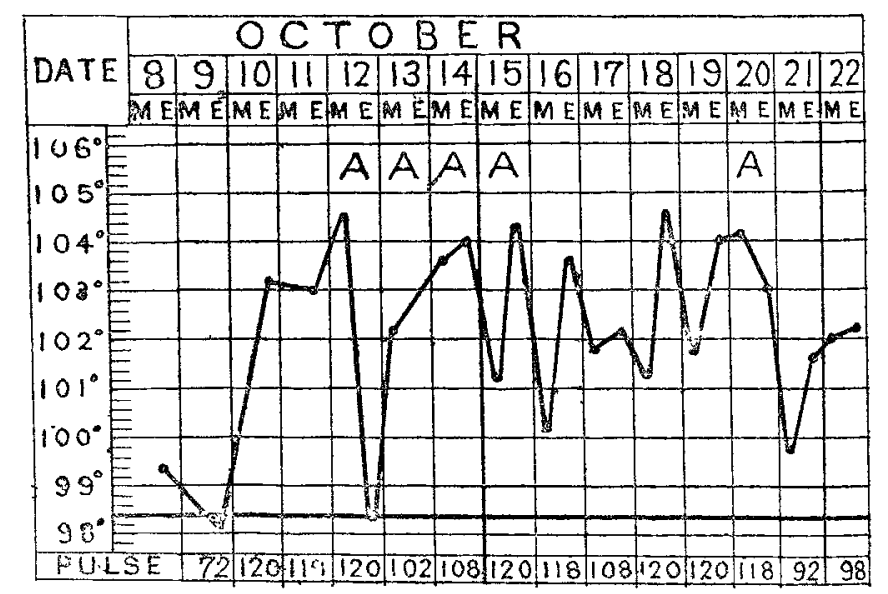

$\Delta$, Ten cubic centimetres of antistreptococcic serum injected.

a fairly normal pulce tracing was taken. The fundus was one finger's breadth above the umbilicus and three fingers' breadth to the right. In the afternoon she complained of pain and the nurse gave her castor oil in the evening. On the 10th there was a motion of the bowels. Headache was complained of and there was pain in the lower part of the abdomen with marked tympanites. The fundus was half finger's breadth above the umbilicus and one finger's breadth to the right. The tongue was brown and furred 